\title{
LOS EFECTOS DE LA INNOVACIÓN EN EL RENDIMIENTO DE LAS MIPYMES \\ DE AGUASCALIENTES: UNA EVIDENCIA EMPÍRICA
}

Gonzalo Maldonado Guzmán Universidad Autónoma de Aguascalientes

Antonia Madrid Guijarro

Universidad Politécnica de Cartagena, España

María del Carmen Martínez Serna

Universidad Autónoma de Aguascalientes

Luis Aguilera Enríquez

Universidad Autónoma de Aguascalientes 



\section{INTRODUCCIÓN}

La innovación permite a las empresas lograr ventajas competitivas sostenibles en el tiempo (Vermeulen, 2004) y representan un elemento determinante del crecimiento económico (Cheng \& Tao, 1999). En este sentido, la innovación y la estrategia son elementos esenciales para mantener e incrementar el nivel de competitividad de las organizaciones (Chandler, 1962; Ansoff, 1965). Por ello, la innovación posibilita que la organización pueda responder rápida y eficazmente a los cambios que requiere el mercado, e incluso permite la posibilidad de adelantarse a dichos cambios y mantener su posición competitiva.

Por otro lado, la innovación es necesaria para ampliar el ciclo de vida de los productos y para tomar ventaja de las nuevas oportunidades que presenta el mercado (Pisano \& Wheelwright, 1995; Bakema et al., 2002; O’Regan et al., 2005). Sin embargo, es demasiado complejo la implementación de un proceso de innovación, especialmente en las Pymes, ya que tienen demasiadas limitaciones en sus recursos financieros, humanos y tecnológicos (Hadjimanolis, 1999), lo que frena los procesos de innovación, principalmente en el desarrollo de nuevos productos y servicios (Larsen \& Lewis, 2007).

Ante la cuestión de si la empresa innovadora genera un mayor beneficio, no existe un claro consenso en la literatura. Geroski y Machin (1992) sugieren que la innovación en $I+D$ provoca un aumento en la rentabilidad y crecimiento de la empresa. Si la innovación implica una mejora del producto y una reducción en los costos, la empresa podrá aumentar sus beneficios y su cuota de mercado. Sin embargo, otros autores sugieren que la innovación, al menos en el corto plazo, puede llevar implícitos bajos beneficios. Por ello la innovación tiende a incrementar el crecimiento y la eficiencia de las organizaciones, pero no inmediatamente un mayor beneficio (Heunks, 1998), debido a los altos costos de la innovación.

Ante esta situación, en el ámbito de la Pyme, una mayor comprensión de la relación entre innovación y rendimiento puede ayudar a que las Pymes desarrollen políticas que mejoren su productividad y competitividad (Freel, 2000; Van Auken et al., 2008). Bajo estos lineamientos y considerando el entorno de un país en vías de desarrollo, como es el caso de México, el objetivo de este trabajo es analizar los efectos que ejerce la innovación en el rendimiento de las Pymes de Aguascalientes. Por lo tanto la pregunta de investigación que intentamos contestar es la siguiente: ¿Está afectando positivamente la innovación de la PYME de Aguascalientes al rendimiento de la misma?

Para ello se llevó a cabo un estudio empírico con una muestra de 400 Pymes pertenecientes a distintos sectores de la actividad industrial. En este estudio, 
la innovación se ha considerado en sentido amplio tomando en cuenta en los análisis el efecto de la innovación en productos, procesos y sistemas de gestión. Asimismo, tal y como utilizan Van Auken et al. (2008) en un estudio sobre los efectos de la innovación en el rendimiento de la Pyme española, consideramos como variable de rendimiento el constructo propuesto por Quinn y Rohrbaugh (1981), el cual distingue diferentes dimensiones del rendimiento tales como: el sistema abierto, los procesos internos, el sistema racional y los recursos humanos.

Los resultados de este estudio son importantes tanto para los gerentes de las Pymes de México, como para las autoridades gubernamentales para que diseñen mejor las políticas económicas encaminadas a fomentar la innovación en las Pymes. Los gerentes obtendrán un mayor conocimiento que cómo influye la innovación en las diferentes dimensiones del rendimiento, de tal manera que lo puedan guiar en la aplicación de uno u otro tipo de innovación, lo que puede optimizar su decisión de inversión. La administración pública podrá identificar el tipo de innovación más beneficioso para las Pymes, elemento que le permitirá el diseño de políticas que estimulen la innovación en las Pymes.

El resto del trabajo se ha organizado de la siguiente manera: en la segunda sección se lleva a cabo la revisión de la literatura y el marco teórico. A continuación se exponen las características de la muestra, las técnicas de recolección de la información, la definición de las variables y su medición, y los modelos estimados. En la cuarta sección se analizan los resultados de las estimaciones y, finalmente, en la quinta sección se resumen los principales hallazgos en las conclusiones.

\section{REVISIÓN DE LA LITERATURA}

El desarrollo del modelo del trabajo de investigación describe la relación de la innovación y el rendimiento de las Pymes. En este sentido, en las siguientes secciones se intentará clarificar los distintos componentes del modelo, a fin de sustentar los planteamientos y resultados obtenidos.

\subsection{Innovación y Rendimiento}

Actualmente existe un claro reconocimiento entre la mayoría de los investigadores que trabajan en innovación, de que este tópico es un factor esencial que facilita la ventaja competitiva e impacta en el crecimiento de las naciones y empresas (Storey, 2000; Galia \& Legros, 2004; Tourigny \& Le, 2004). Por su parte, Freel (2000) concluyó que la innovación es el elemento fundamental por medio del cual las Pymes puedan mantener y mejorar su nivel de crecimiento. En este sentido, la innovación puede ser considerada como un factor estratégico de crecimiento de las empresas (Geroski et al., 1993; Heunks, 1998; Cefis \& Marsili, 2006). 
Por un lado, para que las empresas puedan aplicar la innovación requieren de cambios sustanciales tanto en los factores internos como en los externos a la organización. Si estos cambios se realizan, permitirá a las Pymes obtener un impacto positivo en su crecimiento (Genus \& Coles, 2006), de lo contrario le generará un impacto negativo a la organización (Borgelt \& Falk, 2007). Los trabajos de Geroski y Machin (1992), Moore (1995), Regev (1998), Heunks (1998), Verona (1999), Hughes (2001), Hsueh y Tu (2004), van Auken et al. (2008) y Madrid-Guijarro et al. (2009) proporcionan evidencia empírica de la relación entre innovación y rendimiento de las Pymes.

Por otro lado, la innovación puede ser considerado como un factor esencial que puede proporcionar un alto rendimiento en un creciente número de industrias (Zahra et al., 1999), y en particular puede fortalecer la ventaja competitiva de las empresas (Mone et al., 1998; O'Regan et al., 2005) y proveer un alto rendimiento financiero (Zahra et al., 2000). Asimismo, Keizer et al. (2002) consideraron que la innovación contribuye al crecimiento económico y es el factor más importante por medio del cual las Pymes pueden obtener una ventaja competitiva. Ello debido a que las Pymes tienen la habilidad de desarrollar una innovación más efectiva y desarrollar nuevos productos más rápidamente que las grandes empresas (Storey, 2000; Vossen, 1998). Sin embargo, Harrison y Watson (1998) consideraron que existe una pequeña duda de que las Pymes sean capaces de implementar una innovación efectiva. Aún así, diversas Pymes tienen más oportunidades y ventajas que las grandes empresas, tales como la flexibilidad de personalizar los productos a los requerimientos de los consumidores (O’Regan et al., 2005).

Bajo este contexto, la importancia de la innovación como un factor que proporciona una ventaja competitiva ha sido ampliamente documentada en la literatura empresarial (Grant, 1991; Peteraf, 1993; Barney, 1997; Shoham \& Fieganbaum, 2002; O'Regan et al., 2005; Larsen \& Lewis, 2007; van Auken et al., 2008; Madrid-Guijarro et al., 2009). Asimismo, investigaciones previas muestran cómo la innovación impacta positivamente en el rendimiento de las empresas (Romer, 1994; Grossman \& Helpman, 1994; Barro \& Sala-I-Martin, 1995; Banbury \& Mitchell, 1995; Moore, 1995; Roper et al., 1996; Roper, 1997; Shafer \& Frenkel, 2005; O'Regan et al., 2005; van Auken et al., 2008; Madrid-Guijarro et al., 2009).

Regev (1998) y Cainelli et al. (2004) concluyeron que las empresas que realizaron más innovaciones (innovadoras), mejoraron significativamente su productividad laboral y tuvieron mayores ventas que aquellas empresas que no realizaron ninguna innovación (no innovadoras). Por su parte Hughes (2001), en un estudio realizado en las Pymes de Gran Bretaña, llegó a la conclusión de que las empresas que más innovaron lograron incrementar significativamente sus márgenes de utilidad. De igual manera, Hsueh y Tu (2004) demostraron que la innovación tiene 
efectos positivos en el rendimiento de las Pymes de Taiwán. Por su parte Olav y Leppälahti (1997), en un estudio realizado a las Pymes de Noruega con más de 50 empleados, concluyeron que las Pymes más innovadoras obtuvieron un mayor rendimiento que aquellas empresas no innovadoras.

En este mismo sentido, Yamin et al. (1999) examinaron la relación entre la innovación organizacional y el rendimiento en las Pymes de Australia y llegaron a la conclusión de que las empresas más innovadoras tienen un mayor rendimiento que aquellas menos innovadoras. Asimismo, van Auken et al. (2008) analizaron la relación entre la innovación y el rendimiento en las Pymes de España y demostraron que la innovación impacta positivamente en el desempeño de las empresas, tanto en las industrias de baja tecnología como en las industrias de alta tecnología.

Finalmente, el modelo de la efectividad organizacional de Quinn y Rohrbaugh (1983) ha sido utilizado en este estudio como base para medir el rendimiento de las Pymes. La validación de este modelo ha sido evidente en diversos trabajos de investigación (Walton \& Dawson, 2001; Frankel et al., 2001; Brockman \& Morgan, 2003; Miron et al., 2004; Van Auken et al., 2008). Este modelo proporciona un método comúnmente utilizado para medir la relación entre la cultura organizacional y la innovación; ya que la innovación implica un complejo sistema en el cual intervienen diversos factores. En este sentido, el control interno, las relaciones con los clientes y los recursos humanos juegan un rol importante en el aseguramiento y sostenibilidad de los procesos de innovación. Por ello, Brockman y Morgan (2003) hicieron énfasis en la importancia de conocer a la organización como una importante capacidad para lograr una ventaja competitiva.

A pesar de los numerosos estudios empíricos publicados y de una amplia discusión teórica sobre la innovación y el rendimiento, aún no ha concluido la perspectiva teórica sobre la innovación (Drazin \& Schoonhoven, 1996). En una amplia revisión de la literatura sobre los trabajos de investigación en innovación, Shoham y Fieganbaum (2002) sugieren la necesidad de trabajar en una integración teórica entre el contexto organizacional y la dinámica del nivel industrial, justamente los elementos analizados en este estudio.

Considerando la revisión de la literatura realizada planteamos la siguiente hipótesis de investigación.

La innovación afecta positivamente a las PYMEs de Aguascalientes, de forma que las empresas más innovadoras se beneficiaran de un mejor rendimiento en sus diferentes dimensiones. 


\section{METODOLOGíA}

Para validar la relación entre la innovación y el rendimiento se llevó a cabo una investigación empírica en las Pymes de Aguascalientes. En una primera fase del estudio se aplicó un "Panel Empresarial", en el cual participaron los responsables de las dependencias gubernamentales vinculadas a las Pymes, gestores de instituciones financieras y empresarios de las Pymes, el cual permitió testar el cuestionario que se utilizaría en la segunda fase del estudio.

\subsection{Diseño de la Muestra y Recolección de los Datos}

La población objeto de estudio de este trabajo se obtuvo del Directorio Empresarial 2008 del Sistema de Información Empresarial de México (SIEM) del Estado de Aguascalientes (8,661 empresas registradas hasta agosto del mismo año), considerando únicamente a aquellas empresas que tuvieran entre 5 y 250 trabajadores (MiPymes), el directorio empresarial se redujo a un total de 1,342 empresas. La muestra se seleccionó por medio de un muestreo aleatorio simple con un error máximo del $\pm 4 \%$ y un nivel de confiabilidad del $96 \%$. Los datos se recolectaron por medio de entrevistas personales con los gerentes de las Pymes, y se aplicaron durante los meses de Septiembre a Diciembre de 2008. Estas entrevistas se organizaron a través de la guía de un cuestionario previamente testado por un panel de expertos.

Finalmente, se llevaron a cabo 408 entrevistas durante el año 2008, y de ellas 8 fueron eliminadas por no cumplir con los requisitos establecidos, quedando un total de 400 encuestas validadas, de tal forma que la tasa de respuesta fue del $80 \%$. La encuesta recolectó la información sobre las características de las empresas, las actividades de innovación en los dos años anteriores, y la evolución de determinados ítems que identifican el rendimiento de la empresa.

En la Tabla 1 se muestran los porcentajes de cobertura de la población total considerando tanto el sector de actividad (industria, servicios-comercio y construcción), como el tamaño empresarial a partir del número de empleados. De las 400 empresas de la muestra total, 228 son microempresas, 134 son pequeñas empresas y 38 son empresas medianas. 
Tabla 1

Cobertura de la muestra por sector y tamaño empresarial

\begin{tabular}{|lccc|}
\hline & Población & Muestra & Cobertura \\
\hline Industria & 423 & 190 & $44,9 \%$ \\
Construcción & 72 & 57 & $79,2 \%$ \\
Servicio y Comercio & 847 & 153 & $18,1 \%$ \\
\hline TOTAL & 1342 & 400 & $29,8 \%$ \\
\hline & Población & Muestra & Cobertura \\
\hline Microempresas (6 a & 758 & 228 & $30,1 \%$ \\
10 trabajadores) & & & \\
Pequeñas & 455 & 134 & $29,5 \%$ \\
Medianas & 129 & 38 & $29,5 \%$ \\
\hline Total & 1342 & 400 & $29,8 \%$ \\
\hline Fuente: Elaboración Propia & & & \\
\hline
\end{tabular}

La Tabla 2 muestra de forma más desagregada la pertenencia de la muestra a los distintos sectores de actividad.

Tabla 2

Distribución sectorial de la muestra

\begin{tabular}{|lccccc|}
\hline Sector de actividad & $\begin{array}{c}\text { Micro de } \\
\mathbf{4} \text { a 10 } \\
\text { empleados }\end{array}$ & $\begin{array}{c}\text { Pequeña } \\
\mathbf{1 1} \text { a 50 } \\
\text { empleados }\end{array}$ & $\begin{array}{c}\text { Mediana } \\
\mathbf{5 1} \text { a 250 } \\
\text { empleados }\end{array}$ & $\begin{array}{c}\text { Número } \\
\text { de } \\
\text { empresas }\end{array}$ & Total \\
\hline Servicios & 52 & 21 & 3 & 76 & 19 \\
\hline Comercio & 54 & 20 & 3 & 77 & 19.3 \\
\hline Textil y confección & 31 & 11 & 5 & 47 & 11.8 \\
\hline Mueble & 24 & 16 & 4 & 44 & 11 \\
\hline Agroindustria & 31 & 11 & 6 & 48 & 12 \\
\hline Metalmecámica & 14 & 23 & 4 & 41 & 10.3 \\
\hline Construcción & 17 & 27 & 13 & 57 & 14.3 \\
\hline TICs & 5 & 5 & 0 & 10 & 2.5 \\
\hline Total & & & & 400 & 100 \\
\hline Fuente: Elaboración Propia & & & & & \\
\hline
\end{tabular}

\subsection{Variables de Innovación}

El concepto de innovación es demasiado amplio, la definición más aceptada y compartida por la OCDE y EUROSTAT es la expuesta en el Manual de Oslo: "Las innovaciones comprenden los nuevos productos y procesos, así como las modificaciones tecnológicas importantes de los mismos. Una innovación se considera como tal cuando es introducida en el mercado (innovaciones de productos) o utilizadas en un proceso de producción (innovaciones de procesos). En ellas intervienen toda clase de actividades científicas, tecnológicas, de organización, financieras y comerciales". 
La innovación en productos se materializa en la comercialización de un nuevo artículo o en la mejora de otro ya existente, y la innovación en procesos dota a las empresas de nuevos bienes de equipo o de nuevos procesos de producción. Esta clase de innovación tenderá a incrementar la productividad de las empresas y a reducir sus costos (AECA, 1995). La innovación en métodos de gestión recoge el conjunto de novedades y cambios introducidos en la estructura organizacional de la empresa como es el caso de la comercialización, financiamiento, organización, etc. (AECA, 1995).

Los gerentes de las MiPymes indicaron si la empresa había realizado algún cambio o mejora en sus productos, procesos o sistemas de gestión en los últimos dos años. La Tabla 3 muestra la composición de cada una de las variables de innovación y verifica la validez de la metodología. La fiabilidad de las escalas queda sustentada por el alfa de Crombach superior a 0.7.

Tabla 3

Variables de Innovación

\begin{tabular}{|c|c|c|}
\hline Tipo de innovación & Variables & Validación de la escala \\
\hline $\begin{array}{l}\text { Innovación en } \\
\text { productos }\end{array}$ & $\begin{array}{l}\text { Cambios o mejoras en productos/ } \\
\text { servicios existentes. Comercialización } \\
\text { de nuevos productos/servicios }\end{array}$ & $\begin{array}{l}\alpha \text { de Cronbach }=0.817 \\
\text { Factorial: } 1 \text { factor } \\
\text { Varianza explicada: } 84.5 \% \\
\text { Sig. Bartlett: } 0.000 \\
\text { KM0: } 0.600\end{array}$ \\
\hline $\begin{array}{l}\text { Innovación en } \\
\text { procesos }\end{array}$ & $\begin{array}{l}\text { Cambios o mejoras en los procesos de } \\
\text { procesos o generación de servicios } \\
\text { Adquisición de nuevos bienes de equipo }\end{array}$ & $\begin{array}{l}\alpha \text { de Cronbach }=0.715 \\
\text { Factorial: } 1 \text { factor } \\
\text { Varianza explicada: } 78 \% \\
\text { Sig. Bartlett: } 0.000 \\
\text { KMO: } 0.600\end{array}$ \\
\hline $\begin{array}{l}\text { Innovación en } \\
\text { gestión }\end{array}$ & $\begin{array}{l}\text { Dirección y gestión } \\
\text { Compras y aprovisionamientos } \\
\text { Comercial/ventas }\end{array}$ & $\begin{array}{l}\alpha \text { de Cronbach }=0.840 \\
\text { Factorial: } 1 \text { factor } \\
\text { Varianza explicada: } 76 \% \\
\text { Sig. Bartlett: } 0.000 \\
\text { KMO: } 0.712\end{array}$ \\
\hline \multicolumn{3}{|c|}{$\begin{array}{l}\text { Pregunta del cuestionario: } \\
\text { ¿Ha realizado algún cambio o mejora en sus productos, procesos o sistemas de gestión en los } \\
\text { últimos } 2 \text { años? } \\
\text { NO SI }\end{array}$} \\
\hline
\end{tabular}




\subsection{Variables de Rendimiento}

En el contexto mexicano es extremadamente difícil obtener datos financieros de las empresas, y mucho más de las micro, pequeñas y medianas. De hecho, al contrario de los que ocurre en la economía española, los estados contables de las empresas mexicanas no son accesibles a terceros. En este contexto, la opacidad informativa de la empresa es de gran calado. Ante esta situación, en este trabajo hemos optado por un enfoque subjetivo para medir el rendimiento. En este enfoque, el gerente de la empresa es la pieza fundamental, puesto que su visión sobre cuáles han sido los avances de la empresa puede influir en la gestión futura.

Para elaborar las variables que identifiquen el rendimiento de la empresa utilizamos el modelo de efectividad organizativa de Quinn y Rohrbaugh (1983), que considera cuatro enfoques claves para la empresa: 1 ) Procesos Internos: formalización y controles internos que aseguran el uso efectivo de los recursos; 2) Sistema Abierto: flexibilidad de las relaciones con el entorno y adaptación a las nuevas necesidades del mismo; 3) Sistema Racional: logro de los objetivos planeados e índices de productividad; y 4) Recursos Humanos: enfatiza el bienestar, la motivación y el compromiso de los trabajadores. Este modelo ha sido ampliamente validado en investigaciones previas (Frenkel et al., 2001; Walton \& Dawson, 2001; Brockman \& Morgan, 2003; Miron et al., 2004).

Los gerentes indicaron la evolución de doce ítems (tres ítems para cada enfoque) en su empresa en los últimos años, utilizando para ello una escala tipo Likert de cinco puntos: $1=$ Evolución muy desfavorable a 5 = Evolución muy favorable. Para identificar la evolución en cuanto a sus procesos internos, utilizamos conceptos relacionados con la calidad del producto, la eficiencia de los procesos operativos y la organización de las tareas. Los ítems relacionados con el enfoque de sistema abierto hacen alusión a la satisfacción de los clientes, la rapidez de adaptación a las necesidades de los mercados y la imagen de la empresa.

El rendimiento relativo al sistema racional lo consideramos a partir del incremento en la cuota de mercado, rentabilidad y productividad. Finalmente, el enfoque de recursos humanos se midió a partir de la motivación de los trabajadores, la reducción de la rotación del personal y la reducción del ausentismo laboral. La Tabla 4 muestra la composición de cada una de las variables de rendimiento y verifica la validez de la metodología. La fiabilidad de las escalas queda sustentada por alfas de Crombach superiores a 0.7. 
Tabla 4

Variables de rendimiento

\begin{tabular}{|c|c|c|}
\hline Tipo de enfoque & Variables & Validación de la escala \\
\hline Procesos Internos & $\begin{array}{l}\text { Calidad del producto/servicio } \\
\text { Eficiencia de los procesos operativos } \\
\text { Organización de las tareas del personal }\end{array}$ & $\begin{array}{l}\alpha \text { de Cronbach }=0.874 \\
\text { Factorial: } 1 \text { factor } \\
\text { Varianza explicada: } 80 \% \\
\text { Sig. Bartlett: } 0.000 \\
\text { KM0: } 0.722\end{array}$ \\
\hline Sistema Abierto & $\begin{array}{l}\text { Satisfacción de los clientes } \\
\text { Rapidez de adaptación a las necesidades } \\
\text { de los mercados } \\
\text { Imagen de la empresa y de sus } \\
\text { productos/servicios }\end{array}$ & $\begin{array}{l}\alpha \text { de Cronbach }=0.839 \\
\text { Factorial: } 1 \text { factor } \\
\text { Varianza explicada: } 76 \% \\
\text { Sig. Bartlett: } 0.000 \\
\text { KM0: } 0.723\end{array}$ \\
\hline Sistema Racional & $\begin{array}{l}\text { Incremento de la cuota de Mercado } \\
\text { Incremento de la rentabilidad } \\
\text { Incremento de la productividad }\end{array}$ & $\begin{array}{l}\alpha \text { de Cronbach }=0.895 \\
\text { Factorial: } 1 \text { factor } \\
\text { Varianza explicada: } 83 \% \\
\text { Sig. Bartlett: } 0.000 \\
\text { KM0: } 0.745\end{array}$ \\
\hline Recursos Humanos & $\begin{array}{l}\text { Motivación/satisfacción de los trabajadores } \\
\text { Reducción de la rotación de personal } \\
\text { Reducción del ausentismo laboral }\end{array}$ & $\begin{array}{l}\alpha \text { de Cronbach }=0.823 \\
\text { Factorial: } 1 \text { factor } \\
\text { Varianza explicada: } 74 \% \\
\text { Sig. Bartlett: } 0.000 \\
\text { KM0: } 0.663\end{array}$ \\
\hline
\end{tabular}

\subsection{Modelos}

Con ánimo de analizar si la innovación llevada a cabo en las MiPymes de Aguascalientes ha provocado mejoras en el rendimiento de las empresas, se estiman los siguientes modelos considerando, tanto los distintos tipos de innovación (productos, procesos y sistemas de gestión) como las distintas dimensiones del rendimiento de la empresa (4 enfoques).

$\mathrm{Y}_{\mathrm{i}}=\mathrm{b}_{\mathrm{o}}+\mathrm{b}_{1}$ Familiari $+\mathrm{b}_{2}$ Tamaño $_{\mathrm{i}}+\mathrm{b}_{3}$ Edadi $+\mathrm{b}_{4}$ InProducto $_{\mathrm{i}}+\mathrm{b}_{5}$ Sector $_{\mathrm{i}}+\ldots+$ $\mathrm{b}_{\mathrm{n}-1}$ Sector $_{\mathrm{n}-1}+\varepsilon_{\mathrm{i}}$

$\mathrm{Y}_{\mathrm{i}}=\mathrm{b}_{\mathrm{o}}+\mathrm{b}_{1}$ Familiar $_{\mathrm{i}}+\mathrm{b}_{2}$ Tamaño $_{\mathrm{i}}+\mathrm{b}_{3}$ Edad $_{\mathrm{i}}+\mathrm{b}_{4}$ InProceso $_{\mathrm{i}}+\mathrm{b}_{5}$ Sector $_{\mathrm{i}}+\ldots+$ $\mathrm{b}_{\mathrm{n}-1}$ Sector $_{\mathrm{n}-1}+\varepsilon_{\mathrm{i}}$

$\mathrm{Y}_{\mathrm{i}}=\mathrm{b}_{\mathrm{o}}+\mathrm{b}_{1}$ Familiar $_{\mathrm{i}}+\mathrm{b}_{2}$ Tamaño $_{\mathrm{i}}+\mathrm{b}_{3}$ Edad $_{\mathrm{i}}+\mathrm{b}_{4}$ InGestión $_{\mathrm{i}}+\mathrm{b}_{5}$ Sector $_{\mathrm{i}}+\ldots+$ $\mathrm{b}_{\mathrm{n}-1}$ Sector $_{\mathrm{n}-1}+\varepsilon_{\mathrm{i}}$ 
Donde:

\begin{tabular}{|c|c|}
\hline $\mathrm{Y}_{\mathrm{i}}$ & $\begin{array}{l}=\text { Variables de rendimiento de la Pyme considerando las } 4 \text { dimen- } \\
\text { siones }\end{array}$ \\
\hline Familiar $_{i}$ & $\begin{array}{l}=\text { Variable dicotómica que toma valor } 1 \text { cuando el control mayorita- } \\
\text { rio de la empresa es familiar y o en el resto de casos. }\end{array}$ \\
\hline Tamaño $_{i}$ & $\begin{aligned}= & \text { Variable dicotómica que toma valor } 1 \text { cuando la empresa tiene } \\
& \text { entre } 51 \text { y } 250 \text { trabajadores y o cuando su tamaño es menor. }\end{aligned}$ \\
\hline $\operatorname{Edad}_{i}$ & $\begin{aligned}= & \text { Variable dicotómica que toma valor } 1 \text { cuando la empresa tiene } \\
& \text { más de } 15 \text { años de antigüedad (madura) y o en el resto de casos. }\end{aligned}$ \\
\hline InProducto & $\begin{aligned}= & \text { Variable dicotómica que toma valor } 1 \text { cuando la empresa ha rea- } \\
& \text { lizado cambios o mejoras en sus productos y/o ha comercializado } \\
& \text { nuevos productos y o en el resto de casos. }\end{aligned}$ \\
\hline InProceso $_{i}$ & $\begin{aligned}= & \text { Variable dicotómica que toma valor de } 1 \text { cuando la empresas ha } \\
& \text { realizado cambios o mejoras en sus procesos y/o ha adquirido } \\
& \text { nuevos bienes de equipo y o en el resto de los casos. }\end{aligned}$ \\
\hline InGestión $_{i}$ & $\begin{array}{l}\text { V Variable dicotómica que toma valor de } 1 \text { cuando al empresa ha } \\
\text { realizado cambios o mejoras en sus sistemas de dirección y ges- } \\
\text { tión y/o en compras y aprovisionamientos y/o en comercial-ven- } \\
\text { tas y o en el resto de los casos. }\end{array}$ \\
\hline Sector $_{i}$ & $\begin{aligned}= & \text { Variables dicotómicas que identifican al sector de actividad. En el } \\
& \text { modelo son incluidas } \mathrm{n}-1 \text { variables, siendo la categoría oculta la } \\
& \text { relacionada con el sector de las TICs. }\end{aligned}$ \\
\hline
\end{tabular}

\section{RESULTADOS}

La Tabla 5 muestra los resultados de las estimaciones de los modelos previamente explicados, examinando las relaciones entre las cuatro medidas de rendimiento (procesos internos, sistemas abiertos, sistema racional y recursos humanos), y los tres tipos de innovación (innovación en productos, innovación en procesos e innovación en sistemas de gestión).

Tabla 5

Relación entre el rendimiento y la innovación $(n=850)$

\begin{tabular}{|l|l|c|c|c|}
\hline Enfoque & Variables & $\begin{array}{c}\text { Innovación en } \\
\text { productos }\end{array}$ & $\begin{array}{c}\text { Innovación en } \\
\text { procesos }\end{array}$ & $\begin{array}{c}\text { Innovación en } \\
\text { gestión }\end{array}$ \\
\cline { 3 - 5 } & & Coeficientes & Coeficientes & Coeficientes \\
\hline \multirow{4}{*}{$\begin{array}{l}\text { Procesos } \\
\text { internos }\end{array}$} & Familiar & 0.061 & 0.053 & 0.052 \\
& & $(1.183)$ & $(1.017)$ & $(1.004)$ \\
\cline { 2 - 5 } & & $0.095^{\star}$ & $0.115^{\star *}$ & $0.123^{\star *}$ \\
\cline { 2 - 5 } & Edad & $(1.771)$ & $(2.126)$ & $(2.294)$ \\
\hline & & 0.006 & 0.01 & -0.001 \\
& $(0.123)$ & $(0.017)$ & $(-0.024)$ \\
\hline
\end{tabular}

(tabla 5, continúa) 


\begin{tabular}{|c|c|c|c|c|}
\hline \multirow{7}{*}{$\begin{array}{l}\text { Sistema } \\
\text { abierto }\end{array}$} & $\begin{array}{l}\text { Tipo de } \\
\text { innovación }\end{array}$ & $\begin{array}{l}0.179 * \star \star \\
(3.521)\end{array}$ & $\begin{array}{l}0.094^{\star \star} \\
(1.870)\end{array}$ & $\begin{array}{c}0.066 \\
(1.312)\end{array}$ \\
\hline & & $\begin{array}{l}\mathrm{F}=3.661^{* \star *} \\
\mathrm{R}^{2} \text { Ajustado }=0.068\end{array}$ & $\begin{array}{l}\mathrm{F}=2.792^{\star \star *} \\
\mathrm{R}^{2} \text { Ajustado }=0.047\end{array}$ & $\begin{array}{l}\mathrm{F}=2.622^{* * *} \\
\mathrm{R} 2 \text { Ajustado }=0.043\end{array}$ \\
\hline & Familiar & $\begin{array}{c}0.075 \\
(1.440)\end{array}$ & $\begin{array}{c}0.068 \\
(1.310)\end{array}$ & $\begin{array}{c}0.068 \\
(1.294)\end{array}$ \\
\hline & Tamaño & $\begin{array}{c}0.049 \\
(0.900)\end{array}$ & $\begin{array}{c}0.064 \\
(1.180)\end{array}$ & $\begin{array}{c}0.071 \\
(1.319)\end{array}$ \\
\hline & Edad & $\begin{array}{c}0.014 \\
(0.277)\end{array}$ & $\begin{array}{c}0.011 \\
(0.208)\end{array}$ & $\begin{array}{l}0.009 \\
(0.174)\end{array}$ \\
\hline & $\begin{array}{l}\text { Tipo de } \\
\text { innovación }\end{array}$ & $\begin{array}{l}0.141^{* * *} \\
(2.734)\end{array}$ & $\begin{array}{c}0.077 \\
(1.521)\end{array}$ & $\begin{array}{c}0.051 \\
(1.016)\end{array}$ \\
\hline & & $\begin{array}{l}\mathrm{F}=2.580^{\star \star} \\
\mathrm{R}^{2} \text { Ajustado }=0.042\end{array}$ & $\begin{array}{l}\mathrm{F}=2.086^{\star *} \\
\mathrm{R}^{2} \text { Ajustado }=0.029\end{array}$ & $\begin{array}{l}F=1.963^{*} \\
R^{2} \text { Ajustado }=0.026\end{array}$ \\
\hline \multirow{5}{*}{$\begin{array}{l}\text { Enfoque } \\
\text { racional }\end{array}$} & Familiar & $\begin{array}{c}-0.005 \\
(-0.090)\end{array}$ & $\begin{array}{c}-0.012 \\
(-0.233) \\
\end{array}$ & $\begin{array}{c}-0.003 \\
(-0.055)\end{array}$ \\
\hline & Tamaño & $\begin{array}{l}0.130^{* \star} \\
(2.473) \\
\end{array}$ & $\begin{array}{l}0.147^{\star \star} \\
(2.802)\end{array}$ & $\begin{array}{l}0.141^{\star \star \star} \\
(2.732)\end{array}$ \\
\hline & Edad & $\begin{array}{c}-0.042 \\
(-0.837)\end{array}$ & $\begin{array}{l}-0.046 \\
(-0.908)\end{array}$ & $\begin{array}{l}-0.046 \\
(-0.929)\end{array}$ \\
\hline & $\begin{array}{l}\text { Tipo de } \\
\text { innovación }\end{array}$ & $\begin{array}{l}0.127 \\
(2.544)^{\star \star}\end{array}$ & $\begin{array}{l}0.045 \\
(0.908)\end{array}$ & $\begin{array}{l}0.151^{\star \star \star} \\
(3.148)\end{array}$ \\
\hline & & $\begin{array}{l}F=5.386^{\star \star \star} \\
R^{2} \text { Ajustado }=0.108\end{array}$ & $\begin{array}{l}\mathrm{F}=4.802^{\star \star \star} \\
\mathrm{R}^{2} \text { Ajustado }=0.095\end{array}$ & $\begin{array}{l}F=5.739 * \star \star \\
R^{2} \text { Ajustado }=0.116\end{array}$ \\
\hline \multirow{5}{*}{$\begin{array}{l}\text { Recursos } \\
\text { humanos }\end{array}$} & Familiar & $\begin{array}{c}-0.028 \\
(-0.541)\end{array}$ & $\begin{array}{c}-0.028 \\
(-0.540)\end{array}$ & $\begin{array}{c}-0.027 \\
(-0.533)\end{array}$ \\
\hline & Tamaño & $\begin{array}{c}0.068 \\
(1.263) \\
\end{array}$ & $\begin{array}{c}0.067 \\
(1.259)\end{array}$ & $\begin{array}{c}0.072 \\
(1.362) \\
\end{array}$ \\
\hline & Edad & $\begin{array}{c}0.026 \\
(0.506)\end{array}$ & $\begin{array}{c}0.026 \\
(0.503)\end{array}$ & $\begin{array}{l}0.024 \\
(0.476)\end{array}$ \\
\hline & $\begin{array}{l}\text { Tipo de } \\
\text { innovación }\end{array}$ & $\begin{array}{c}0.046 \\
(0.909)\end{array}$ & $\begin{array}{c}0.063 \\
(1.273)\end{array}$ & $\begin{array}{c}0.052 \\
(1.064)\end{array}$ \\
\hline & & $\begin{array}{l}\mathrm{F}=3.960^{\star \star \star} \\
\mathrm{R}^{2} \text { Ajustado }=0.075\end{array}$ & $\begin{array}{l}\mathrm{F}=4.040^{\star * \star} \\
\mathrm{R}^{2} \text { Ajustado }=0.077\end{array}$ & $\begin{array}{l}\mathrm{F}=3.991^{* * *} \\
\mathrm{R}^{2} \text { Ajustado }=0.076\end{array}$ \\
\hline \multicolumn{5}{|c|}{$\begin{array}{l}\left({ }^{\star}\right): p<0,1 ;\left({ }^{\star *}\right): p<0,05 ;\left({ }^{\star \star \star}\right): p<0,01 \\
\text { Las estimaciones se han realizado incluyendo variables dummies sectoriales } \\
\text { Coeficientes: coeficientes estandarizados (en paréntesis el valor de la t ) } \\
\text { FIV: Factor de inflación de la varianza }\end{array}$} \\
\hline
\end{tabular}

\subsection{Efectos de la innovación sobre el sistema de procesos internos}

Las estimaciones referentes al sistema de procesos internos evidencian coeficientes positivos y significativos para dos de los tres tipos de innovación considerados. Los tres modelos muestran estadísticos de ajuste significativos demostrando la validez global de los modelos $(\mathrm{F}=3.661 ; \mathrm{F}=2.792 \mathrm{y} \mathrm{F}=2.622$, 
respectivamente). La única variable de control significativa es el tamaño, teniendo asociado un signo positivo. El mayor tamaño de la empresa provoca un incremento en su rendimiento en cuanto a eficacia y eficiencia interna, puesto que la variable dependiente es la dimensión de rendimiento de procesos internos.

La innovación en productos y la innovación en procesos tienen asociados coeficientes positivos y estadísticamente significativos. Este resultado revela que la mejora de productos existentes o la introducción de un nuevo producto provoca mejoras en la eficiencia interna de la empresa. De igual manera, y como era de esperar, mejoras en los procesos ya existentes o la adquisición de nuevos procesos desembocan en un incremento en la eficiencia de la Pyme vía procesos internos. Por el contrario, la innovación en los sistemas de gestión no provoca ninguna influencia significativa sobre la dimensión de rendimiento de procesos internos de la Pyme de Aguascalientes.

\subsection{Efectos de la innovación sobre el sistema abierto}

Las estimaciones del modelo cuya variable dependiente es la dimensión del rendimiento de sistema abierto, evidencian coeficientes positivos y significativos para uno de los tres tipos de innovación considerados. Los tres modelos muestran estadísticos de ajuste significativos, demostrando con ello la validez global de los modelos ( $\mathrm{F}=2.580 ; \mathrm{F}=2.086$ y F $=1.963$, respectivamente), principalmente por las variables de control de carácter categórico incluidas para tomar en cuenta el sector de actividad al que pertenece la empresa.

La innovación en productos tiene asociado un coeficiente positivo y estadísticamente significativo. Este resultado revela que la mejora de productos existentes o la introducción de un nuevo producto provoca mejoras en el rendimiento de la empresa en cuanto a su capacidad para satisfacer a la clientela, rapidez para adaptarse a las necesidades de los mercados y su mejor imagen como empresa y sus productos. Por el contrario, las innovaciones en procesos y en sistemas de gestión no provocan ninguna influencia significativa sobre la dimensión de rendimiento de sistema abierto de la Pyme de Aguascalientes.

\subsection{Efectos de la innovación sobre el sistema racional}

Las estimaciones relativas al sistema racional revelan coeficientes positivos y significativos para dos de los tres tipos de innovación considerados. Los tres modelos muestran estadísticos de ajuste significativos, demostrando la validez global de los modelos ( $F=5.386 ; F=4.802$ y $F=5.739$, respectivamente). La única variable de control significativa es el tamaño, teniendo asociado un signo positivo. Por lo tanto, tal y como ocurría en la estimación cuya variable dependiente era el proceso interno, el mayor tamaño de la empresa provoca un incremento de su rendimiento en cuanto a incremento de la cuota de mercado, rentabilidad y pro- 
ductividad, puesto que la variable dependiente es la dimensión de rendimiento de sistema racional.

La innovación en productos y la innovación en sistemas de gestión tienen asociados coeficientes positivos y estadísticamente significativos. Este resultado postula que la mejora de productos existentes o la introducción de un nuevo producto provocan mejoras en la rentabilidad de la empresa. Por el contrario, la innovación en procesos no provoca ninguna influencia significativa sobre el rendimiento medido por indicadores de rentabilidad y productividad de la Pyme de Aguascalientes.

\subsection{Efectos de la innovación sobre el sistema de recursos humanos}

Las estimaciones relativas al sistema de recursos humanos revelan coeficientes positivos, pero no significativos para los tres tipos de innovación considerados. Los tres modelos muestran estadísticos de ajuste significativos, demostrando la validez global de los modelos ( $\mathrm{F}=3.960 ; \mathrm{F}=4.040$ y $\mathrm{F}=3.991$, respectivamente), como consecuencia de las variables de control de carácter categórico incluidas para tomar en cuenta el sector de actividad al que pertenece la empresa. La falta de significatividad ligada a las variables de innovación, evidencian la escasa integración y participación del personal en las actividades de innovación. Este resultado muestra una clara debilidad de las Pymes de Aguascalientes.

\section{CONCLUSIONES}

El objetivo principal de este trabajo es analizar el efecto que provocan los distintos tipos de innovación en el rendimiento de las Pymes de Aguascalientes. La definición de innovación utilizada es bastante amplia abarcando innovación en productos, innovación en procesos e innovación en sistemas de gestión. Las variables de rendimiento utilizadas no son cuantitativas, sino que se basan en la percepción de los gerentes a través del modelo de Quinn y Rohrbaugh (1981), metodología también utilizada recientemente por Van Auken et al. (2008). La innovación y sus efectos es un tema de investigación que ha recibido una elevada atención en los últimos años desde ámbitos académicos y profesionales. Esta atención se debe a que la innovación se presenta como la pieza clave que determina la competitividad y, por ende, la supervivencia de la empresa.

En el contexto mexicano, este tipo de trabajos son necesarios puesto que México se encuentra en una posición de debilidad en cuanto a inversiones en innovación, tanto pública como privada, en comparación con sus competidores globales. La muestra utilizada en este estudio consta de 400 MiPymes localizadas en el Estado de Aguascalientes, que han llevado a cabo o no algún tipo de innovación. La información se recogió a través de una entrevista al gerente o propietario 
de la Pyme, guiada con un cuestionario. Los análisis revelan que las empresas que llevaron a cabo innovaciones obtuvieron mejores resultados que aquellas empresas que no innovaron.

Concretamente, las empresas que innovaron en cuanto a sus productos obtuvieron una mayor eficiencia (procesos internos), son más capaces de satisfacer a sus clientes y adaptarse a sus necesidades (procesos internos), elevan su rentabilidad y adaptabilidad (sistema racional). Las empresas que innovaron en procesos mejoraron su eficiencia en la organización de tareas (procesos internos). Las empresas que innovaron en cuento a sistemas de gestión, mejoraron su posición en cuento a cuota de mercado y rentabilidad (sistema racional).

Por consiguiente, el tipo de innovación que influye en un mayor número de dimensiones de rendimiento es la innovación en productos. Por otra parte, es necesario remarcar que ningún tipo de innovación está afectando al ámbito de recursos humanos. Este resultado puede estar evidenciando una debilidad muy importante, puesto que puede estar promovido por la falta de integración e implicación del personal de la empresa en el diseño de los proyectos innovadores.

Los resultados de este estudio son útiles tanto para los gerentes de las Pymes mexicanas, como para los diseñadores de las políticas económicas. Por una parte, los gerentes obtendrán un mayor conocimiento de cómo está influyendo las innovaciones en las diferentes dimensiones del rendimiento empresarial, lo que puede guiar el realizar un tipo de innovación u otro, pudiendo optimizar sus decisiones de inversión. Por otra parte, la administración pública puede identificar el tipo de innovación que está siendo más beneficioso para las Pymes, punto que le puede llevar al diseño de políticas que estimulen estas inversiones, o el análisis de por qué algunos tipos de innovación no están funcionando en la Pyme de Aguascalientes. 


\section{REFERENCIAS}

Ansoff, H. (1965), Corporate Strategy: an analytical approach to business policy for growth and expansion, McGraw-Hill, New York.

Bakema, H.G., Baum, J.A.C. and Mannix, E.A. (2002), "Management challenges in a new time”, Academy of Management Journal, Vol. 45 No. 5, pp. 916930.

Banbury, C. and Mitchel, W. (1995), "The effect of introducing important incremental innovations on market share and business survival", Strategic Management Journal, No. 16 (Special Issue), pp. 161-182.

Barney, J. (1997), Gaining and Sustaining Competitive Advantage, AddisonWesley, Reading, MA.

Barro, R. and Sala-I-Martin, X. (1995), Economic Growth, McGraw-Hill, New York.

Borgelt, K. y Falk, I. (2007), "The leadership/management conundrum: innovation or risk management", Leadership \& Organizational Development Journal, Vol. 28, pp. 122-136.

Brockman, B. and Morgan, R. (2003), "The role of existing knowledge in new product innovativeness and performance", Decision Science, Vol. 34 No. 2, pp. 385-420.

Cainelli, G., Evangelista, R. and Savona, M. (2004), "The impact of innovation on economic performance in services", The Service Industries Journal, Vol. 24 No. 1, pp. 116-130.

Cefis, E. y Marsili, O. (2006), "Survivor: the role of innovation in firm's survival", Research Policy, Vol. 35, pp. 626-641.

Chandler, A.D. (1962), Strategy and Structure, Massachusetts Institute of Technology Press.

Cheng, L. y Tao, Z. (1999), "The impact of public policies on innovation and imitation: the role of R\&D technology in growth models", International Economic Review, Vol. 40 No. 1, pp. 187-207.

Freel, M., (2000), "Barriers to product innovation in small manufacturing firms", International Small Business Journal, Vol. 18 No. 2, pp. 60-79. 
Frenkel, A., Shafer, D., Koschalzky, K. and Walter, G. (2001), "Firm characteristics, location and regional innovation: a comparison between Israeli and German industrial firm”, Regional Studies, Vol. 35 No. 5, pp. 413-427.

Galia. F. y Legros. D. (2004), "Complementarities between obstacles to innovation: evidence from France”, Research Policy, Vol. 33, pp. 1185-1199.

Genus, A. y Coles, A. (2006), "Firm strategies for risk management in innovations", International Journal of Innovation Management, Vol. 10, pp. 113-126.

Geroski, P. and Machin, S. (1992), "Do innovating firms outperform non-innovator?”, Business Strategy Review, Vol. 14, pp. 79-90.

Geroski. P., Machin, S. y Van Reenen, J. (1993), "The profitability of innovations firms”, RAND Journal of Economics, Vol. 24 No. 2, pp. 198-211.

Grant, R. (1991), "The resource-based theory of competitive advantage: implications for strategy formulation", California Management Review, Vol. 33 No. 3, pp. 114-135.

Grossman, G. and Helpman, E. (1994), "Endogenous innovation in the theory of growth”, Journal of Economic Perspective, Vol. 8 No. 1, pp. 23-44.

Hadjimanolis, A. (1999), "Barriers to innovation for SME in a small less developed country (Cyprus)", Technovation, Vol. 19, pp. 561-570.

Harrison, N.J. and Watson, T. (1998), The focus for innovation in small and medium service enterprises, Conference Proceedings of Western Decision Sciences Institute, 27th Annual Meeting, Reno

Heunks. F. (1998), "Innovation, creativity and success", Small Business Economics, Vol. 10, pp. 263-272.

Hsueh, L. and Tu, Y. (2004), "Innovation and the operational performance of newly established small and medium enterprises in Taiwan", Small Business Economics, Vol. 23, pp. 99-113.

Hughes, A. (2001), "Innovation and business performance. Small entrepreneurial firms in the UK and the US", New Economy, Vol. 8 No. 3, pp. 157-163. 
Kaizer, J., Dijkstra, L. and Halman, J. (2002), "Explaining innovative efforts of SMEs. An exploratory survey among SMEs in the mechanical and electrical engineering sector in Netherlands", Technovation, Vol. 22 No. 1, pp. $1-13$.

Larsen, P. and Lewis, A. (2007), "How award-winning SMEs manage the barriers to innovation", Creativity and Innovation Management, Vol. 16 No. 2, pp. 142-151.

Madrid-Guijarro, A., Garcia-Perez-de-Lema, D. y Van Auken, H. (2009), "Barriers to innovation among Spanish manufacturing SMEs", Journal of Small Business Management, aceptado pendiente de publicación.

Miron, E., Erez, M. and Naveh, E. (2004), "Do personal characteristics and cultural values that promote innovation, quality and efficiency compete or complement each other?", Journal of Organizational Behavior, Vol. 25 No. 2, pp. 175-200.

Mone, M.A., McKinley, W. and Bargar, V.L. (1998), "Organizational decline and innovation: a contingency framework", Academy of Management Review, Vol. 32, pp. 115-132.

Moore, B. (1995), What Differentiates Innovative Small Firms?, Innovation Paper No. 4, ESRC Centre for Business Research, University of Cambridge, England.

O’Regan, N., Ghobadian, A. and Sims, A. (2005), "Fast tracking innovation in manufacturing SMEs", Technovation, Vol. 25 No. 1, pp. 1-11.

Olav, S. and Leppälahti, A. (1997), "Innovation, firm profitability and growth", Paper provide by The Step Group, Studies in Technology, Innovation and Economic Policy in Step Report Series No. 1997/01.

Peteraf, M. (1993), "The cornerstones of competitive advantage: a resource-based view", Strategic Management Journal, Vol. 14 No. 3, pp. 179-191.

Pisano, G.P. and Wheelwright, S.C. (1995), "The new logic of high tech R\&D", Harvard Business Review, Vol. 73 No. 5, pp. 93-105.

Quinn, R. and Rohrbaugh, J. (1981), "A competing values approach to organizational effectiveness", Public Productivity Review, Vol. 5, pp. 122-140. 
Quinn, R. and Rohrbaugh, J. (1983), "A spatial model of effectiveness criteria: towards a competing values approach to organizational analysis", Management Science, Vol. 29 No. 3, pp. 363-377.

Regev, H. (1998), "Innovation, skilled labor, technology and performance in Israeli industrial firms", Economy Innovation and New Technology, Vol. 5, pp. 301-323.

Romer, P. (1994), “The origins of endogenous growth", Journal of Economic Perspective, Vol. 8 No. 1, pp. 3-22.

Roper, S. (1997), "Product innovation and small business growth: a comparison of strategies of German, UK and Irish companies", Small Business Economics, Vol. 9 No. 6, pp. 523-537.

Roper, S., Love, J., Dunlop, S., Ashcroft, B., Hofmann, H. and Vogler-Ludwig, K. (1996), Product Innovation and Development in UK, German and Irish Manufacturing, Northern Ireland Economic Research Centre, Queens University, Kingston, Ontario, Canada.

Shafer, D. and Frenkel, A. (2005), "R\&D, firm size and innovation: an empirical analysis”, Technovation, Vol. 25, pp. 25-32.

Shoham, A. and Fieganbaum, A. (2002), "Competitive determinants of organizational risk-taking attitude: the role of strategic reference points”, Management Decision, Vol. 40 No. 2, pp. 127-141.

Storey, J., (2000), “The management of innovation problem", International Journal of Innovation Management, Vol. 4 No. 3, pp. 347-369.

Tourigny, D. y Le, C. (2004), "Impediments to innovation faced by Canadian firms", Economics of Innovation and New Technology, Vol. 13 No. 3, pp. 217-250.

Van Auken, H., Madrid-Guijarro, A. y García-Pérez-de-Lema, D. (2008), "Innovation and performance in Spanish manufacturing SMEs, International Journal of Entrepreneurship and Innovation Management, Vol. 8 No. 1, pp. 36-56.

Vermeulen, P. (2004), "Managing product innovation in financial service firms", European Management Journal, Vol. 22 No. 1, pp. 43-50. 
Verona, G. (1999), “A resource-based view of product development”, Academy of Management Review, Vol. 24 No. 1, pp. 132-142.

Vossen, R. (1998), "Relative strengths and weaknesses of small firms in innovation”, International Small Business Journal, Vol. 16 No. 3, pp. 88-94.

Walton, E.J. and Dawson, S. (2001), "Managers' perceptions of criteria of organizational effectiveness", Journal of Management Studies, Vol. 38 No. 2, pp. 173-199.

Yamin, S., Gunasekaran, A. and Mavondo, F.T. (1999), "Innovation index and its implications on organizational performance: a study of Australian manufacturing firms", International Journal of Technology Management, Vol. 17 No. 5, pp. 495-503.

Zahra, S., Ireland, R. and Hitt, M. (2000), “International expansion by new venture firms: international diversity, mode of market entry, technology learning and performance", Academy of Management Journal, Vol. 43 No. 5, pp. 925-950.

Zahra, S.A., Nielsen, A.P. and Bognar, W.C. (1999), "Corporate entrepreneurship, knowledge and competence development", Entrepreneurship: Theory and Practice, Vol. 23 No. 3, pp. 169-189. 